Research Article

\title{
Smart Supply Chain Management Using the Blockchain and Smart Contract
}

\author{
Manoshi Das Turjo (D), ${ }^{1}$ Mohammad Monirujjaman Khan $\mathbb{D}^{1},{ }^{1}$ Manjit Kaur ${ }^{(\mathbb{D}},{ }^{2}$ \\ and Atef Zaguia ${ }^{3}{ }^{3}$ \\ ${ }^{1}$ Department of Electrical and Computer Engineering, North South University, Bashundhara, Dhaka 1229, Bangladesh \\ ${ }^{2}$ School of Engineering and Applied Sciences, Bennett University, Greater Noida, India \\ ${ }^{3}$ Department of Computer Science, College of Computers and Information Technology, Taif University, Taif 21944, Saudi Arabia
}

Correspondence should be addressed to Mohammad Monirujjaman Khan; monirujjaman.khan@northsouth.edu

Received 23 July 2021; Accepted 20 September 2021; Published 28 September 2021

Academic Editor: Kashif Zia

Copyright ( $) 2021$ Manoshi Das Turjo et al. This is an open access article distributed under the Creative Commons Attribution License, which permits unrestricted use, distribution, and reproduction in any medium, provided the original work is properly cited.

\begin{abstract}
The manufacture of raw materials to deliver the product to the consumer in a traditional supply chain system is a manual process with insufficient data and transaction security. It also takes a significant amount of time, making the entire procedure lengthy. Overall, the undivided process is ineffective and untrustworthy for consumers. If blockchain and smart contract technologies are integrated into traditional supply chain management systems, data security, authenticity, time management, and transaction processes will all be significantly improved. Blockchain is a revolutionary, decentralized technology that protects data from unauthorized access. The entire supply chain management (SCM) will be satisfied with the consumer once smart contracts are implemented. The plan becomes more trustworthy when the mediator is contracted, which is doable in these ways. The tags employed in the conventional SCM process are costly and have limited possibilities. As a result, it is difficult to maintain product secrecy and accountability in the SCM scheme. It is also a common target for wireless attacks (reply attacks, eavesdropping, etc.). In SCM, the phrase "product confidentiality" is very significant. It means that only those who have been validated have access to the information. This paper emphasizes reducing the involvement of third parties in the supply chain system and improving data security. Traditional supply chain management systems have a number of significant flaws. Lack of traceability, difficulty maintaining product safety and quality, failure to monitor and control inventory in warehouses and shops, rising supply chain expenses, and so on, are some of them. The focus of this paper is on minimizing third-party participation in the supply chain system and enhancing data security. This improves accessibility, efficiency, and timeliness throughout the whole process. The primary advantage is that individuals will feel safer throughout the payment process. However, in this study, a peer-to-peer encrypted system was utilized in conjunction with a smart contract. Additionally, there are a few other features. Because this document makes use of an immutable ledger, the hacker will be unable to get access to it. Even if they get access to the system, they will be unable to modify any data. If the goods are defective, the transaction will be halted, and the customer will be reimbursed, with the seller receiving the merchandise. By using cryptographic methods, transaction security will be a feasible alternative for recasting these issues. Finally, this paper will demonstrate how to maintain the method with the maximum level of safety, transparency, and efficiency.
\end{abstract}

\section{Introduction}

In today's global market, supply chain management (SCM) is critical. It has a significant influence on the global economy. SCM is often defined as the movement of goods from producer to consumer. It is divided into numerous phases, starting with the supply of raw materials and ending with the client, and includes the producer, distributor, and retailer. It is a global process in which components are sourced from a single location, packaged, and supplied globally. Traditional supply chain management serves a broad goal but falls short of full compliance. Giving the final 
customer the ability to reverse the transaction and assuring the quality of the items supplied has several limits. It usually corresponds to forward flows, or the flow of products from the sender to the recipient $[1,2]$. Supporting the reverse flow of items and transactions for every consumer is also critical. The traditional supply chain management system might be disrupted by blockchain and smart contracts. The supply chain can benefit from the blockchain's transparency and immutability [3]. By offering a secure mechanism for collecting data and developing and running programmed scripts or applications known as smart contracts, the blockchain aids in the modernization of the supply chain [4]. Smart contracts can help supply chain managers track the origin and security of their products. We discussed the issues and came up with a solution.

The approach of developing a conceptual framework for a supply chain management system is included in this research. Its main objective is to leverage blockchain and smart contracts to provide safe transactions and high-quality products. It will allow any client to return a product and receive a refund for the money spent on it, resulting in a trustworthy worldwide market. Most significantly, under our paradigm, the whole SCM system will undergo a significant transformation.

A blockchain is a continuously growing list of logs known as cryptographically connected and secured blocks. The blocks are connected cryptographically. The bulk of the nodes check the blocks in the blockchain network. The block will then be added to the chain that all network nodes share when it has been verified. Handling a single piece of data necessitates thousands of instances, each of which requires a significant amount of work and time to avoid. Access to information is associated with higher quality in various blockchain systems. There are a few qualities that distinguish blockchain from other technologies. Data on the blockchain, for example, is immutable, tamper-resistant, and based on a decentralized network, and it can be hacked and encrypted. In general, three forms of blockchain exist: public or unauthorized, private or permitted, and consortium blockchain. Each one has specific characteristics because of the uniqueness of the network's geographic area $[5,6]$.

According to Nick Szabo, smart contracts are "a computerized transaction protocol that meets contract conditions." Smart contracts are pieces of code (software or scripts) written in a high-level programming language [7] like Java, C++, NodeJS, Python, Go, Solidity, and others. For smart contracts, many blockchain systems employ various high-level programming languages [7]. The Hyperledger manufacturing platform employs the programming languages NodeJS and Python. On the other hand, for their smart contracts, Ethereum employs a sound programming language [8]. Copies of intelligent contracts are available on the blockchain-based network for each peer. Scripts for smart contracts are run automatically, independently, and openly. It is always executed in a secure environment to guarantee that code and data integrity are maintained. $[9,10]$.

The essence of a cryptocurrency is that it may be exchanged through transactions and that it may be added to new blocks and mined by miners in the most secure way possible. There are three major levels in the crypto realm. Technology, currency, and tokens are the three. The coin is bitcoin, and the technology is blockchain (bitcoin is not just a currency). It is a set of rules. A protocol is a collection of rules that govern how individuals interact on a network. It determines how public keys and signatures should be used for authentication in bitcoin, ethereum, and other cryptocurrencies. The Coin is a built-in asset of the protocol that allows player interaction and is used to reward individuals for mining the blockchain and creating blocks. It is also used to let users buy stuff from other people. Smart contracts are used with tokens. There is a distinction to be made between a token and a coin. When someone invests in a coin, they are also investing in the underlying coin's protocol. They are investing in the concept behind what they are building if they invest in a token.

With the passage of time, the supply chain has evolved. As a result of the global market, SCM has become increasingly important in the current world. However, there are still some substantial challenges in supply chain management in numerous areas. Between the buyer and the seller, there must be trust. A trusted environment is required for transaction processing. However, in today's commercial world, there is no such thing as complete trust. In the transaction process, the intermediaries in a supply chain have a great deal of power. They can manipulate market value without telling genuine supply chain members, allowing them to benefit at the expense of the ultimate consumer. In today's supply chain, there is no encrypted mechanism to store people's private information in numerous businesses, hospitals, and other locations. This data will be vulnerable to cyberattacks, exposing the sensitive public and private information. Because of the supply chain's intermediaries, there is no price transparency. This will provide a direct link between the buyer and the vendor, making the transaction more transparent and trustworthy. Commodities flow in only one direction in today's supply chain management system. As a result, if a product is defective, the customer must bear the repercussions. He had no choice but to take the risk. There are several stages where paperwork must be manually filled out. In a global transaction, the monitoring mechanism is also based on humans. It is more susceptible to human mistakes, resulting in unjustified price rises and less chance to track down the source of the problem. As a result of the aforementioned factors, supply chain management has a significant impact on the worldwide market. The market is still shaky. There is no such thing as a flawless competition.

In this paper [11], it was proposed to do research on supply chain risk management finance using blockchain technology. We analyze the causes of their operational risks, trade authenticity risks, payback risks, and contingent risks in conjunction with blockchain technical characteristics and the supply chain finance business model and react to and track data in real time using physical sensors to improve supply chain risk control efficiency. Dwivedi et al. describe in this article [12] how the blockchain mechanism works in tandem with the existing pharmaceutical system to provide a 
more efficient supply chain management method. In the supply chain system, this paper offers a blockchain-based information sharing scheme that is safe and uses intelligent contracts and consensus methods. Using intelligent contract technology, the proposal also includes a way of securely providing the needed cryptographic keys to all parties. Alfonso-Lizarazo et al. investigated the usefulness of reverse logistics, or backward product flows and forward logistics, in the palm oil supply chain [13]. The authors presented a closed-loop system that considers "green operations." In both forward and backward flow, it attempts to maintain the environmental sustainability. The report also explains how statistical methods were used to perform mathematical modeling that resulted in a positive output across the supply chain. However, the implementation at the industrial level and data security and administration are not shown. In this essay, Yuan et al. looked at the supply chain management information system method and blockchain as significant technology [14]. From the perspective of blockchain, the process and consensus collaborative management approach is presented, which improves transaction process management and blockchain system consensus, accounting, and so on. Nehai and colleagues [15] present an innovative technique for smart contract validation. It uses a model-checking approach. The model investigates a number of rules for converting smart contracts into model checks. The method works on three levels to display the nature, logic, and execution of the smart contract. The NuSMV tool is used to develop and run programmed smart contract scripts. It is an excellent technique for confirming smart contracts, but IoT device interoperability and blockchain administration remain issues. Kshetri [16] discussed how blockchain may aid supply chain objectives such as speed, cost, risk, and product quality. It also looked into how IoT devices may be linked into a supply chain system based on blockchain. The application of blockchain technology in well-known supply chain use cases throughout the world, such as Alibaba and Maersk, has been extensively researched. It does not, however, provide the global oil supply chain with a full framework. Chang et al. [17] looked at how the pursuit of transparency and accountability across supply chain processes can potentially influence decentralization and automation. A comparative analysis of the current and proposed frameworks was conducted to support the core reasoning of this study. Ahmed and Dixit [18] have shown that consumers throughout India relied on these Kirana shops during the COVID-19 epidemic, which forced the closure of most other retail outlets. While COVID-19 created a new consumer affiliation for Kirana shops and offered new opportunities for these businesses to increase their client bases and product variety, it also exposed supply chain management flaws. Kamran et al. [19] discussed how he makes suggestions to key players in the logistics operations sector of the logistics business that is interested in using blockchain technology. Apart from the study's methodological limitations, system compatibility and layer configuration issues may cause possible difficulties when scaling up the implementation. Liu and Guo [20] discussed Matlab about the findings that indicate that blockchain technology may help propel the fresh food e-commerce supply chain to a greater level of management, coordination, and integration throughout the whole industrial chain. Investing in the blockchain system within a certain budget range may enhance not only product dependability but also the performance of each major component of the fresh food e-commerce supply chain, as well as overall performance. Yoo and Won [21] talked about a system that applies blockchain and smart contracts to the price-tracking component of supply chain management systems to ensure product distribution structure transparency. By increasing transparency in the SCM, this method enables businesses to monitor their transactions, preventing them from chasing excessive profits. By using Ethereum technology, the suggested reference model solves the shortcomings of current models. Furthermore, the researchers used the model to show its functionality in a real-time setting, which may serve as a model for future study. Compared with others, this paper progressively diminishes the participation of third parties in the supply chain system and makes data more secure. This will make the entire process more accessible, efficient, and time-efficient. Most importantly, individuals will feel more secure during the payment process. This paper has its own cryptocurrency with smart contracts to enable more secure dealings with products and put an end to their trust issues. This e-commerce website was secured in a highly secure way in this paper. Many blockchain papers are available on e-commerce websites. These are either peer-topeer encrypted systems or smart contract embedded systems. In this study, however, it utilized a peer-to-peer encrypted system as well as a smart contract. There are a few other features as well. Because this paper utilized an immutable ledger, the hacker will be unable to get access to our system. Even if they get access to the system, they will be unable to alter any of the data. In the transaction procedure, this paper also utilized blockchain. If the goods are defective, the transaction will be halted and the customer will be reimbursed, with the merchandise being returned to the seller. In addition, the contract will be revised. As a result, the transaction process may be trusted. As a result, purchasers will be less likely to fall prey to fraud or other financial problems. As a result, our technology outperforms currently available e-commerce websites. It is safer and more dependable.

Blockchain and cryptocurrencies, as well as smart contracts, are briefly discussed in the first section. Section 2 gives an overview of the approach and materials utilized and a discussion of the concerns and a review of the literature. Section 3 depicts the design and outcomes of our efforts. The influence of the design is discussed in four sections. Finally, the work that can be done in the future and the conclusions expressed in Section 4 are reviewed.

1.1. Problem Statement. On a daily basis, supply chain management systems encounter problems, and nearly all of them require immediate attention and action. The degree and intricacy of these issues may vary. Easy data access, quality and sustainability, supplier management, and 
managing consumer expectations are just a few of them. The Supply Chain Management system confronts ever-increasing challenges year after year. And, with the emphasis on stability, these concerns are now front of mind. After all, supply chains are at the heart of effective business operations, and problems will inevitably have an influence on a company's bottom line.

1.2. Motivation. Blockchain technology validates and stores data using blockchain data structures, generates and updates data using distributed node consensus methods, uses encryption to guarantee data transmission and access security, and employs intelligent script code. It is a novel computer and distributed infrastructure paradigm for programming and manipulating data.

The transaction data produced by each participating entity is packed into a data block, which is then organized in chronological order to form a chain of data blocks in the blockchain system. The main body has the same data chain and cannot be tampered with unilaterally. Any changes to the information must be approved by a certain percentage of the topic, and only new material may be added. The old data cannot be changed or removed. The identification of each subject and the transaction information between entities are open and transparent and cannot be faked, thanks to information sharing and consistent decision-making.

\section{Method and Methodology}

This section delves into the many methods and materials used to achieve the goal. The modeling approach is based on the concept of the blockchain as a cyber SC chain of information services that represents the operational fulfillment of the physical SC. When transaction activities begin and conclude, the blockchain maintains an account of them. As a result, the operations of logistics companies may be viewed as information services that they provide to the blockchain architecture. Smart contract design, in this sense, may be thought of as the computation of start and completion timings for information services in a blockchain-driven cyber environment that mirrors real SC activities.

2.1. Outline of the Full System. This system proposes an abstract concept after briefly explaining and reflecting on current definitions of smart contracts. Because of the concept's novelty and its sophisticated technological foundation, there is no common definition of smart contracts at this time. Given the lack of agreement on nomenclature, it appears that providing an overview of existing techniques and refining an appropriate description is of the highest relevance.

In Figure 1, the whole structure of the blockchain and smart contract working mechanism is depicted. A variety of logistics service providers can take up some operations in the SC that are progressively arranged as flow-oriented activities (i.e., intermediates, carriers).

Different operations may be assigned to several logistics service providers. Assigning operations to logistics firms will

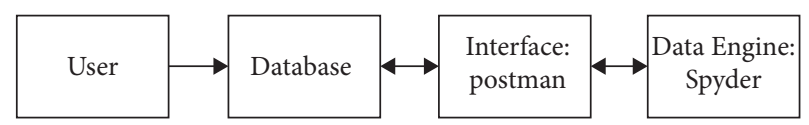

Figure 1: A minimal overview of the complete system.

result in a variety of work lead times and pricing because different logistics service providers operate at different times and at different rates. The process of creating smart contracts includes assigning logistics businesses to jobs and arranging their activities in the contracts, which results in a Blockchain design structure. The start and finish of the execution of the operations of the transaction will be recorded on the blockchain. As a result, the operations of logistics firms might be classified as information services provided by the blockchain architecture. In this sense, smart contract design may be described as the choice to start and stop information services in a blockchain-driven cyberspace that replicates physical SC activities.

For blockchain python, for smart contracts and cryptocurrency purposes, solidity was used in this new supply chain strategy. My ether wallet and Ganache were also used since virtual transactions were performed here for a more realistic outcome. The Spider IDE for implementing Python, the remix IDE for solidity, and nodejs for web pages are used in this system. Figure 2 is the entire process of the system, from the start of the seller's transaction.

Whenever any seller intends to buy any product, he has to do it on the website and there will be no involvement of any third party which can manipulate the payment method. After the buyer gets the product, he will unblock the payment that he has made and then the seller will get his payment. This transaction data will be autoupdated by the website. If, anyhow, the buyer gets a damaged product or becomes dissatisfied with the product he got, then he can return the product and take back his payment through the website. This will not cost any extra money to anyone. After they both make a clear statement review of the transaction, only then will the payment be updated.

Figure 3 is the diagram of the whole mechanism of this system. It will begin with the website receiving data from the user. After the transaction, the customer must approve if the goods are satisfactory or not. The administrator will be able to see the contents of this section.

It will expand in accordance with market circumstances. Any announcement may be made from here. All sales, customer information, and bounce rate data will be managed via this admin panel. The website will automatically update the transaction details. If the customer receives damaged goods or is unhappy with the product, he may return it and get a refund via the website. Nobody will have to pay any additional money as a result of this. Only when they have both completed a thorough statement review of the transaction can the payment be adjusted. Figure 4 is shown. This is the login page for the seller. The homepage features three web pages, which lead to three additional pages.

Another one of them is the login page for the seller. In order to log in, the seller will have to type in his e-mail address and password into his personal account. This page 


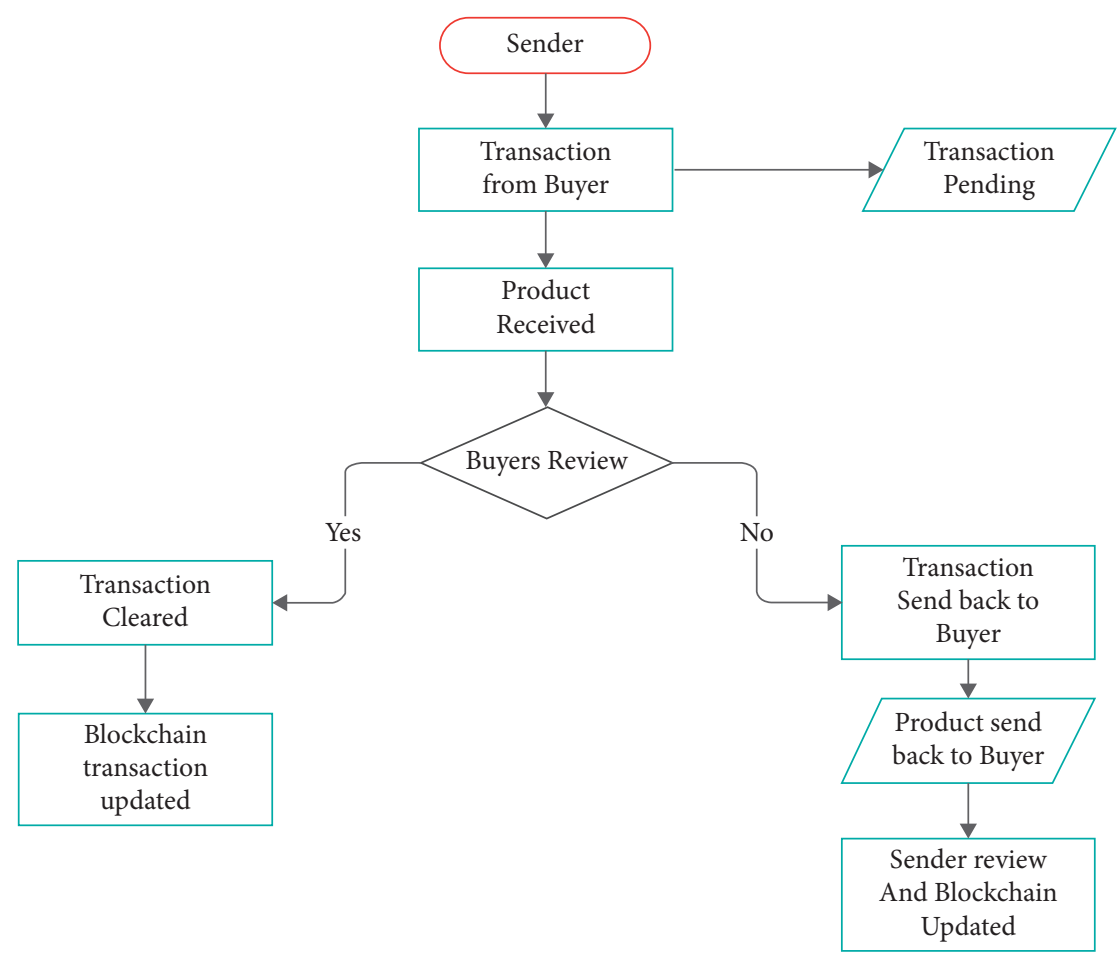

FIgURE 2: The flowchart demonstrating the algorithm used in this SCM.

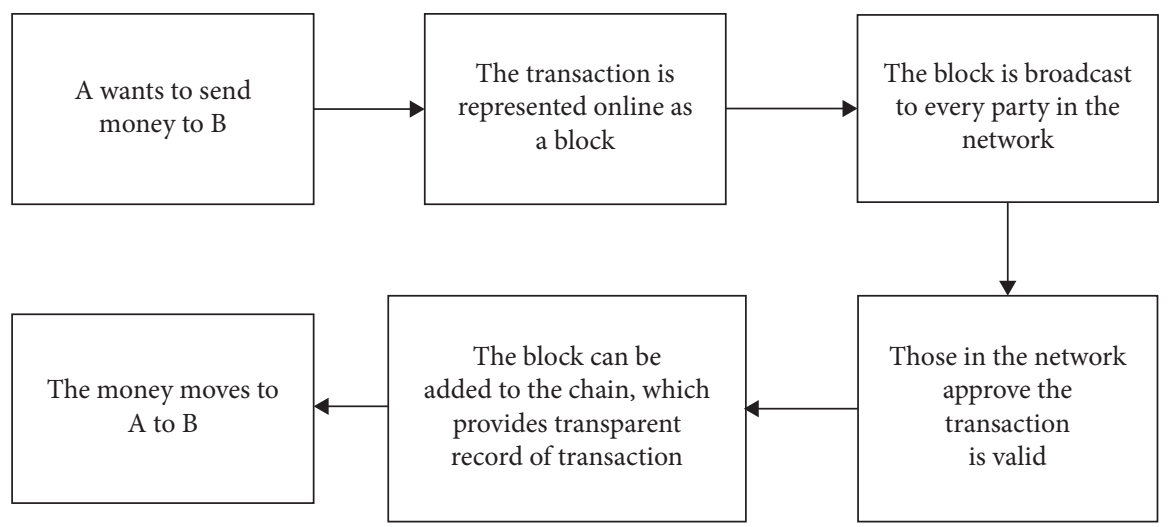

Figure 3: Block diagram of the whole system.

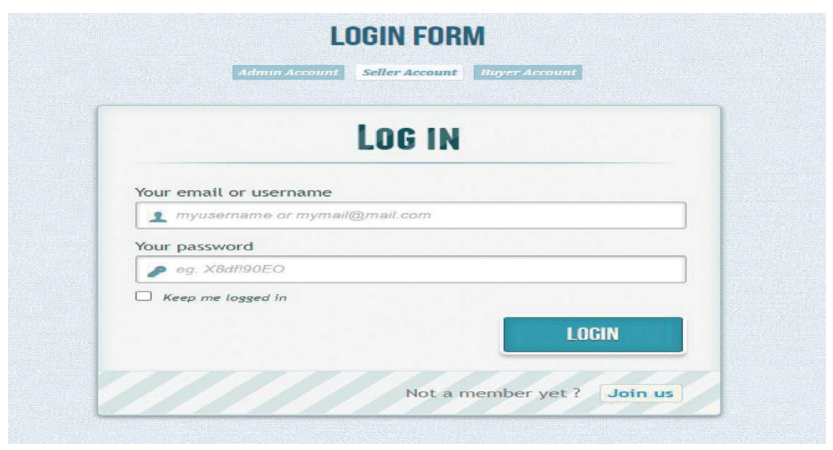

Figure 4: Login page for a seller account. 
allows you to create three different types of accounts. This page's main features are the admin account, seller account, and buyer account. If the user already has an account, the username and password will be required. However, if someone is creating one for the first time, they will need an e-mail address and a password. Every activity may be controlled and monitored by the admin account. The system requires that the seller and buyer accounts follow the same set of rules. For the development of this website system, it required Javascript, CSS, HTML, and PHP. In Figure 5, the registration page for buyers is shown.

The buyer will fill out the registration form with various pieces of information, like their username, e-mail address, and password. They will also type the password they used to confirm it again. The information will be typed and stored in the local database. Using the administrative control panel, it can be seen.

\subsection{Data Security and Transaction in the Blockchain and} Smart Contract. Transactions are not born in the block. When one transaction occurs, the list needs to be empty. Otherwise, it will not get all the separate data. Blocks cannot have the same data in each block. A transaction is put into a block if it is authorized by a majority of the nodes. Each block references the one before it, forming the blockchain. A miner must examine if a transaction is eligible to be processed according to the blockchain history before adding it to their block. The transaction is legitimate and can be included in the block if the sender's wallet balance has sufficient funds according to the current blockchain history. In Figure 6, it is shown that in the traditional system, if we want to buy any big asset, we have to pay for it and, after paying the amount, we get a deed that is the proof of the asset.

If the deed is hacked or manipulated, then the asset owner has no more of their own. It is a volatile way to have ownership, which can be misleading at any time. Someone erases just one line of information, and the asset can lose its license. Client-server networks are used in traditional databases. A user (referred to as a client) may make changes to data that is kept on a central server. The database is still controlled by a specified authority, which verifies a client's credentials before granting access. Because this authority is in charge of database management, the data may be changed or even destroyed if the authority's security is breached. In Figure 7, after using blockchain technology here, we can call it an immutable ledger.

If anyone buys an asset here and keeps all the information in the block, then it is impossible to change the data. Because if any of the data is changed in the block, the whole system gets an alarm as they are all connected with their previous hash number. And it has its own transaction system, which is very secure and trustable. These are the facts that make any traditional ledger an immutable ledger. Public verifiability, provided by integrity and transparency, is a fundamental feature of blockchain technology that differentiates it from conventional database technology.

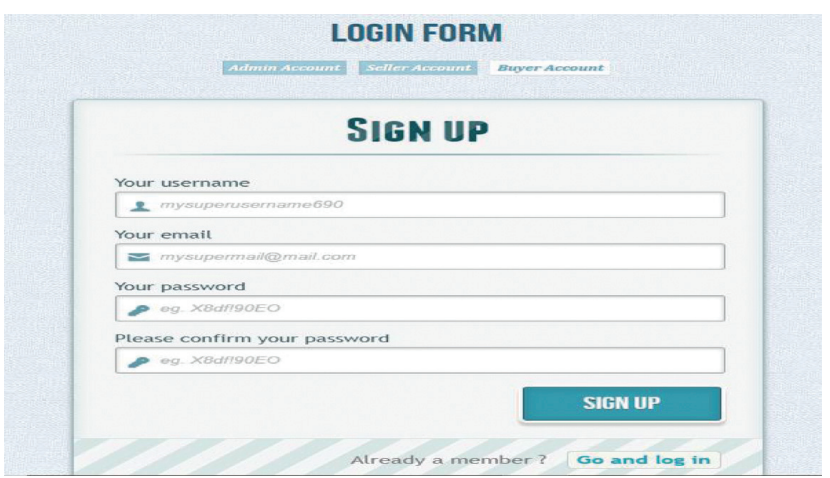

FIgURE 5: Sign-up page for a buyer account.

2.3. Chain and Data Component. This section introduces the basic notion of smart contracts by discussing the nature and types of contracts. From a legal and economic standpoint, we first outline the basic aspects of contracts and their various roles throughout the relationship lifecycle. Then, after examining several ways of defining smart contracts, we offer a broad definition. This part concludes with a critical examination of the role of distributed ledger technology in the idea of smart contracts. In Figure 8, it shows all the functions which have been imported. The date time function is used for each block to have its own timestamp when the block is created and mined. The hashlib function will need to hash the blocks because the hash function will be used here.

Here, using the json function, we will encode the blocks before we hash them. The flask function will be used by the flask library. We will need a flask class because this will use a web application and jsonify, which will take the message and interact with the blockchain with the postman. In the blockchain class, there is a genesis block, a chain function, and a block function that will add a new block and will mine a block. The create_block function will take two arguments: proof and the previous hash number. In Figure 9, proof_of_function has two arguments. One is self, which is for using an instance object that will be created by the class. The other one is previous_proof, for creating a way for miners to solve the problem.

Here, the new_proof value is 1 because after every iteration, it needs to increment by one until it gets the right proof. check_proof will do the checking part to see if it is correct proof or not. The hash_operation contains four leading zeros, which will make mining difficult for the miners, and it is a string of 64 characters. The encode function will encode the string in the right format, which is expected by the sha256 function. In Figure 10, the function of the transaction has been declared. This add_transaction method will conduct the process through the self, sender, and receiver keys of the argument.

This will service the transaction before adding it to the block. This list of data will use the append function to add a new transaction. For every new transaction, it is necessary to add the previous data, which will be done by the previous_blockfunction. Before returning to the previous block function, it will add +1 . As a result, the number will automatically rise, the list will grow, and data will be stored. 


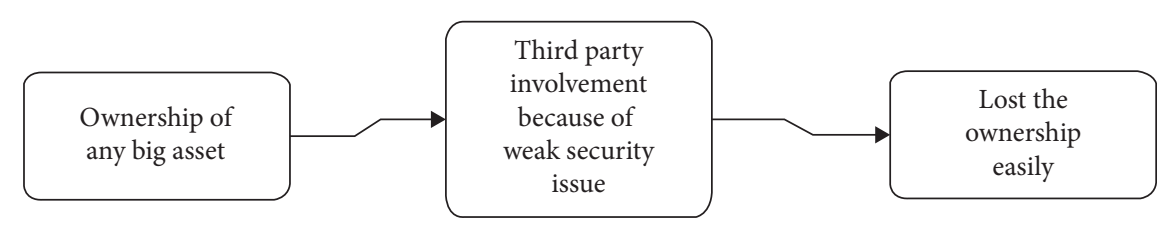

Figure 6: Traditional ledger with an unwanted security issue.

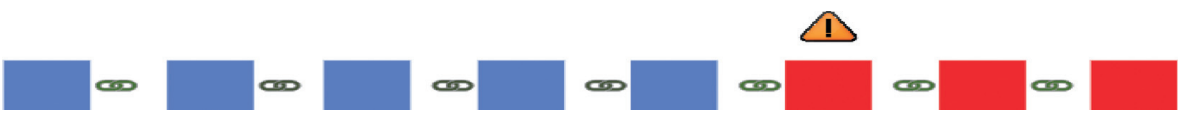

FIGURE 7: Immutable ledger with high security.

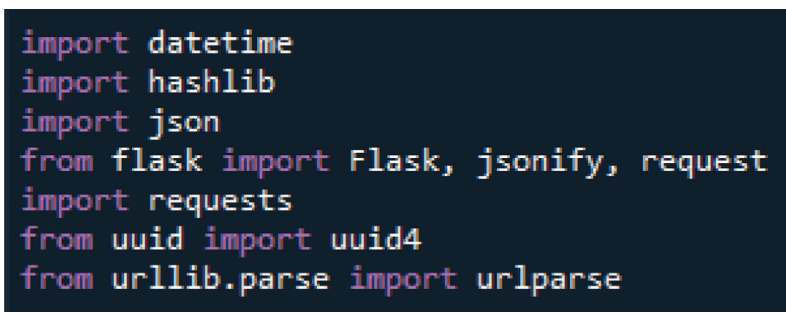

Figure 8: Imported function for the blockchain in spider.

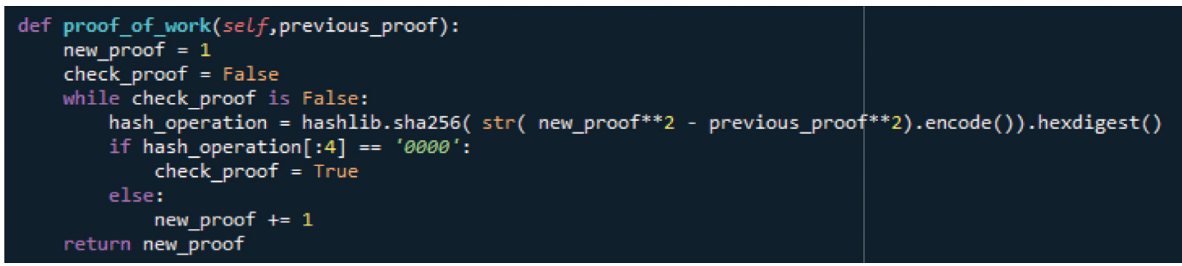

Figure 9: Function for the proof of work in spider.

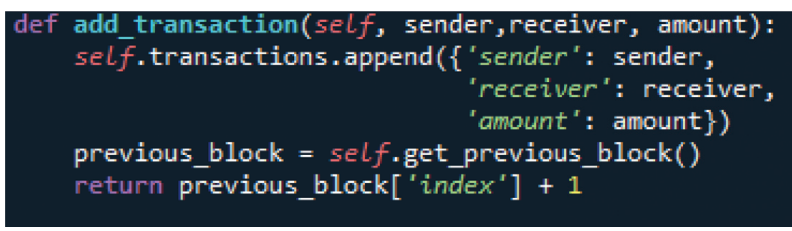

FIGURE 10: Function of transaction in spider.

2.4. Transaction Component. After defining the version of solidity, there will be some functions we need to add to connect with my ether wallet and Ganache. We have declared the total amount that people who will mine can use from here. And after that, the coin value of our created coin named "hadcoin." In Figure 11, the transaction method has been initiated. There will be two mining. One is for equity hadcoins and the other is for equity USD, which is for dollars.

Mapping is like a function, but this data will be stored in an array. It is not like a function that takes a variable and returns it. It is more like an array that will have an input variable which will be the investor's address. It will return the equity in hadcoins and the other will be in USD. In solidity, address is a type of work that works as a function. Modifiers can check if the investor can buy or sell any

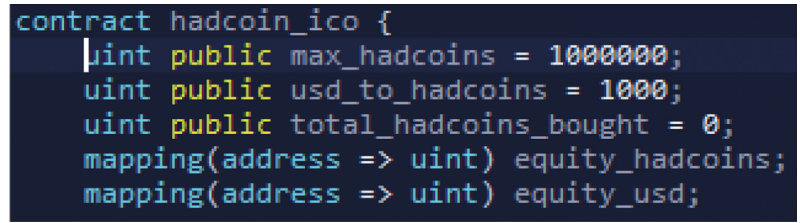

FIGURE 11: Hadcoin creations and adjusting USD in remix.

hadcoins or not. In Figure 12, the control panel for the entire system is shown. All of the transactions that have occurred will be added here automatically. It has the ability to monitor and regulate the operations of miners as well as data.

There will also be information on the buyer and seller, as well as data from Ganache containing transaction details. Details about the buyer's account, the seller's account, and the transaction will be logged here. This section's 


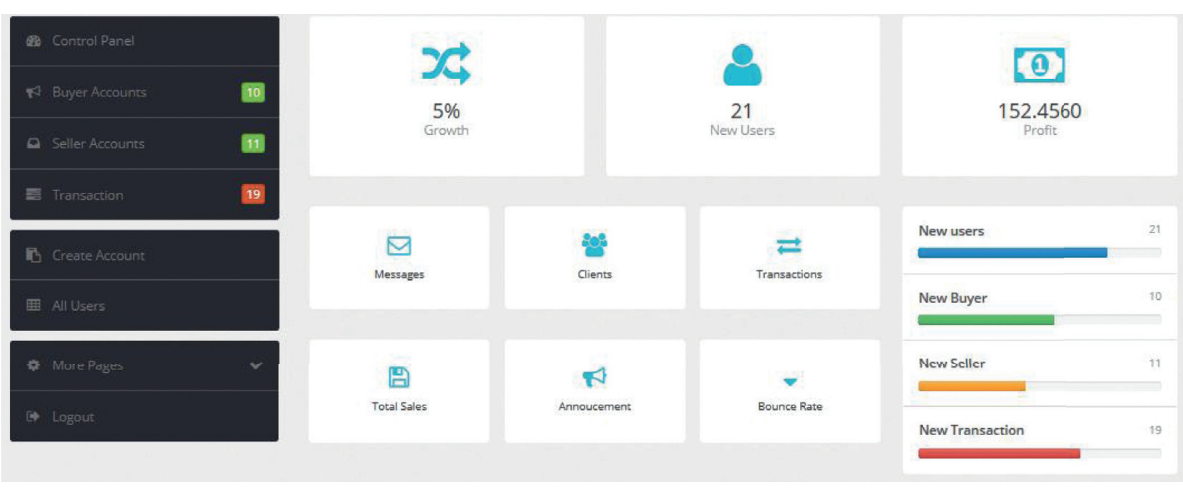

FIGURE 12: Control panel of the website.

information will be visible to the administrator. Its growth will naturally reflect market conditions. From here, an announcement may be issued. This admin panel will manage all sales, customer information, and bounce rate information. New buyers will be able to see the past information of any previous buyers, making this method extremely trustworthy.

\section{Result and Design Analysis}

This is the get_chain output. Here, index means the number of the block, as this is the first block, so it has no previous block. Proof indicates the proof of work, which is different for every single block. A Timestamp indicates when the block was created, the exact time, and date with a number. As it is the first block, no transaction happens here. In Figure 13, this is a random block from all the blocks created for mining. Here the index number is different. It has a previous hash which is the hash value of its previous block.

The proof number is different from the first block, which means mining is happening here. The genesis block is the initial block in any blockchain. As this is the first step in the process, it has no prior hash number. It will include evidence of labor, which will be the same for all blocks. Transactions will be placed here using the "hadcoin" coin. As soon as all of the blocks are added to the system, the length will grow and the system will grow too. It will also contain a timestamp, which is a record of the moment when it was first added to the list. In Figure 14, the fourth block is mined. After mining, it will automatically be added here and will show the message text. The difficulty is always set at a certain time interval and then modified every two weeks, such that a block may be constructed at a predetermined time period. As a result, the difficulty of the network sets a predetermined time period between building two blocks, which is roughly 10 minutes (in Bitcoin).

Previous_hash is the hash number of its immediate previous hash and proof is the unique number of each block. The Timestamp function will show the exact time of when it was mined. The most crucial aspect of this system is mining. This mining is done using a fixed algorithm. Many blocks may be mined at once, but only one will get the proper code and be added to the list. This block will get the prize, while the others will not. The procedure, with the exception of mining, will not be particularly genuine. In Figure 15, a block has been mined and this will be added to the main chain. The admin will get to know that with the message "Congratulations, you just mined a block!," this is a confirmation text for miners, and with this, it will ensure mining. The data is then "hashed" by the node, which converts it into a hash value or "hash," which must always include a specific number of zeros. The node determines if a hash satisfies the difficulty requirements. The hash must begin with the appropriate number of zeroes. If the hash satisfies the difficulty requirements, it is disseminated to the rest of the network's miners. The first miner to discover a valid hash converts the block into a new block and is paid for the block reward and fees.

It will display the index number of 4 for this specific block. A message text that confirms whether or not the block has been mined. If mining does not take place, the output will be negative. Every block has a unique number, which for this block is 21391. This system's timestamp shows when it was mined. There is no other way to control this system; it has its own set of rules that it will adhere to throughout the system. In Figure 16, in the transaction section, the amount indicates how many times the sender sends and the sender receives a separate unique number for each transaction. The first miner has a hexadecimal number of sixty-four digits (a "hash") that exceeds or equals the goal hash. It is pretty much a deviation.

This section depicts what the sender and recipient will get once the virtual transaction is completed. The sender and receiver will be reconnected as a result of this procedure, and since they are both unique, they will not be confused by any other customers. For these two clients, both of these figures are produced. Whenever a blockchain transaction flag is raised, there must be a blockchain consensus to update it on the blockchain. Installed on the blockchain network, members nodes in a blockchain consensus protocol agree on a ledger content and cryptographic hate and digital signatures to guarantee the integrity of transactions instead of depending on third parties to broker transactions. These blockchain transactions, if validated, are deemed successful and irrevocable. Transactions depend a lot on hash and hash values. Figure 17 shows that if it is needed to check if the 


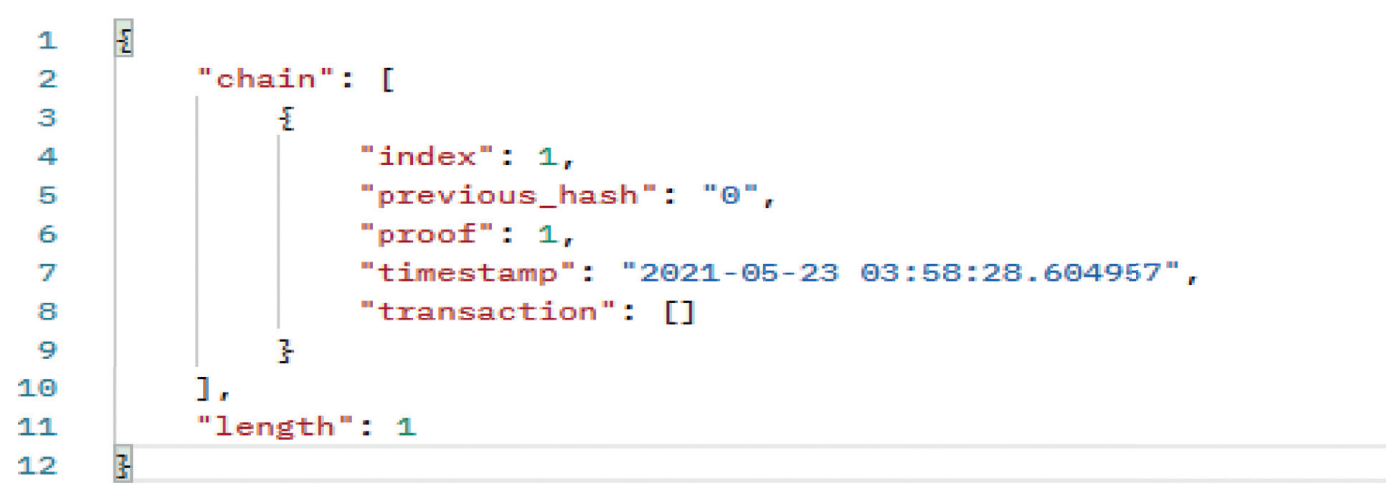

Figure 13: Genesis block and details.

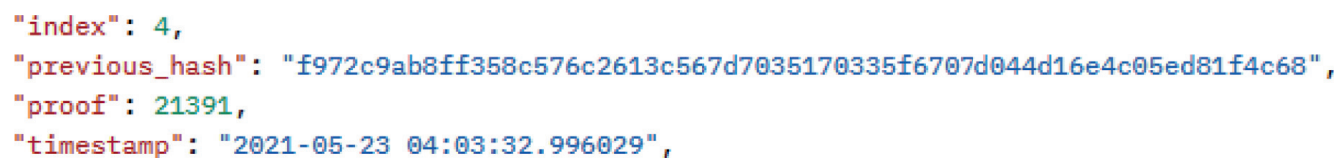

FIGURE 14: Adding blocks in the list.

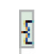

Figure 15: After mining text in postman.

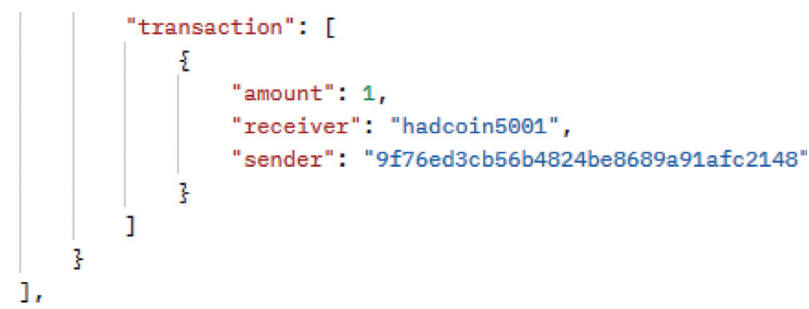

Figure 16: Transaction occurs in postman.

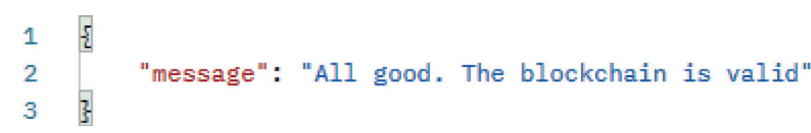

Figure 17: Valid or invalid checking in postman.

blockchain is mining correctly or not, then with the is_valid command, it can be checked. If it stops working, then it will not be valid.

If any sort of unauthentic behavior is detected, the chain will be immediately disrupted. The validation text will not be displayed if this is the case. It will display the phrase "There must be a problem." As a result, the system's working process must be halted, and data must be double checked. As previously said, nothing can be altered manually, and each piece of data will have its own unique identification. This is an advantage of the system. Figure 18 is from Ganache after a transaction occurs in a smart contract.

Before the transaction happened, the balance of ethereum was $100 \mathrm{ETH}$ and the TX COUNT was 0. After the transaction occurs, it becomes $99.97 \mathrm{ETH}$ and the TX COUNT becomes 3 because we have mined three times. Here we need to use its private key code, which is just right after the index. In Figure 19, it is shown that all the transaction data will be stored in the transactions section of Ganache. 


\begin{tabular}{llll}
\hline ADDRESS & BALANCE & TXCOUNT & INDEX \\
$0 \times 2$ E7FeaE564C05F1E3ae78Bac6201d1F95329A744 & 99.97 ETH & 3 & 3 \\
\hline
\end{tabular}

FIgURE 18: Proof of the transaction data in Ganache.

$$
\text { TXHASH }
$$

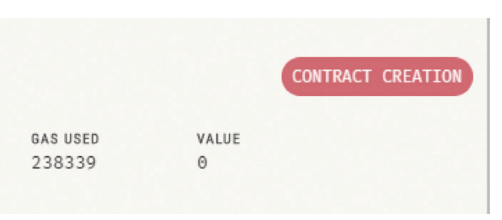

FIgURE 19: Adding the transaction data in Ganache.

\begin{tabular}{|c|c|c|c|}
\hline \multicolumn{4}{|c|}{ Transactions } \\
\hline & Transactions & Status & Timestamp \\
\hline 0 & transaction1 & CLEARED & Date.now0) \\
\hline$\circ$ & transaction2 & CLEARED & Date.now0 \\
\hline 0 & transaction 3 & CLEARED & Date.now0 \\
\hline$\circ$ & transaction 4 & CLEARED & Date.now() \\
\hline 0 & transaction 5 & CLEARED & Date.now() \\
\hline
\end{tabular}

FIGURE 20: Record of all the transactions.

TABLE 1: Comparison table between this paper and other papers.

\begin{abstract}
Points of this paper
(1) Third-party interventions are removed as a result of the deployment of the SCM system described in this article, and stron relationships between peers are formed.

(2) The transaction procedure is transparent and highly secure.

(3) This paper contains the strongest immutable ledger technology to prevent cyberattacks, which results in a safe website.

(4) Makes proper use of a smart contract. As a result, the system is more dependable.

(5) This paper's structured SCM is transparent, and refunds are easy to get if a product is defective.
\end{abstract}

\section{Points of other papers}

(1) Third-party interventions are frequent in SCM management systems since not every paper proposes their own coin as a currency [4].

(2) The transaction system is broken. Both the vendor and the buyer's trust are affected [5].

(3) Absence of an immutable ledger on this other paper. If there is any, then it is not secure enough. As a result, the website was hacked [7].

(4) There is no proper use of smart contracts. As a result, there's a risk that critical information will be tampered with [11].

(5) They provide a very difficult refund procedure. Because of this, many unwanted situations are faced by customers [14].
The address of who has made the transaction, how much he has used, and how many times will be stored in this section. The gas used value will be autogenerated by the ganache website from the data of my_wallet account. This is the most secure way to the transaction by using your own coin through ethereum, and the data are also safe and secure. They all have their own distinct worth that cannot be duplicated. The whole system will be separated and secured as a result of this. Hadcoin's approach provides greater security than the conventional method. In Figure 20, it depicts all of the transaction records.

The image is only viewable by accessing the administrator panel. When a transaction between a seller and buyer is successful, the data for the record will be updated in the administration control panel. Additionally, the date and time of the transaction are displayed. Every transaction is recorded here, and the list will become larger as more transactions are made. Only 5 transactions have been made 
thus far, according to this section. When the status is clear, it indicates that both the buyer and the seller have completed their transaction. The Date now option will provide time information. It contains all of the transaction information.

3.1. Comparison with Other Papers. Table 1 clearly shows the comparison between this paper's materials and the flaws of other papers.

In this article, several very strong security measures have been added, making the system very safe and trustworthy. Other papers, on the other hand, have mostly overlooked these problems, which is why their systems have grown insecure and easy to hack. This paper is up to par since it has an immutable ledger, smart contracts, appropriate transactions, and simple refund and return processes. Every point in previous papers has a flaw. Some of them failed to correctly implement the smart contract, which is the primary cause of the website's failure.

\section{Conclusion}

The goal of this article is to make supply chain management more intelligent, current, and secure. This framework is immutable and tends to give total transaction transparency. It protects our website from unauthorized access and data manipulation. Furthermore, smart contracts cut the amount of time spent on tedious paperwork. In conventional supply chain management, a lot of documentation is usually necessary. The blockchain keeps the information as proof, making smart contracts immutable. The transaction, immutability, and refundable processes in supply chain management are primarily influenced by this paradigm. This study proposed an end-to-end product supply method. It also allows all customers to return a product if they are unhappy with it and receive a refund for their purchase. Every actor's function and role have been specified. It also means that our framework may be used for a variety of reasons. Smart contracts are also discussed in terms of their structure. The difficulties that individuals experienced with old procedures will be permanently eliminated as a result of the findings of this paper. Among its numerous benefits (the most important of which is the capacity to keep data safe), this research promises to speed up and decrease transaction costs, as well as increase financial inclusion by offering more possibilities for people who do not have easy access to financial services.

This paper is a small-scale and extremely particular piece. However, if there is a large volume of data, the latency may be affected. Blockchain transactions will be extremely beneficial in terms of storage and computational costs. Implementing decentralized databases like BigchainDB and HBasechainDB is another way to boost throughput. Furthermore, in the event of large-scale deployment, tracking devices and more actors can be added to the framework. As the amount of data grows, we may employ off-chain architecture to store the original data, and the proof of existence may be retained on the blockchain itself. This might be a potential future research topic for this investigation.

\section{Data Availability}

No data were used to support the findings of this study.

\section{Conflicts of Interest}

The authors declare that there are no conflicts of interest regarding the study.

\section{Acknowledgments}

The authors are thankful for the support from Taif University Researchers Supporting Project (TURSP-2020/114), Taif University, Taif, Saudi Arabia.

\section{References}

[1] C. Prahinski and C. Kocabasoglu, "Empirical research opportunities in reverse supply chains," Omega, vol. 34 , no. 6, pp. 519-532, 2006.

[2] L. Xiaoming and F. Olorunniwo, "An exploration of reverse logistics practices in three companies," Supply Chain Management International Journal, vol. 13, no. 5, pp. 381-386, 2008.

[3] S. Apte and N. Petrovsky, "Will blockchain technology revolutionize excipient supply chain management?" The Journal of Excipients and Food Chemicals, vol. 7, no. 3, pp. 76-78, 2016.

[4] S. Alqahtani, X. He, R. Gamble, and M. Papa, "Formal verification of functional requirements for smart contract compositions in supply chain management systems," in Proceedings of the Hawaii International Conference On System Sciences, pp. 5278-5287, Maui, USA, January 2020.

[5] O. Alfandi, S. Otoum, and Y. Jaraweh, "Blockchain solution for IoT based critical infrastructures: byzantine fault tolerance," in Proceedings of the 2020 IEEE Network Operations and Management and Symposium, pp. 1-4, Budapest, Hungary, April 2020.

[6] D. Magazzeni, P. Mcburney, and W. Nash, "Validation and verification of smart contracts: a research agenda," Computer, vol. 50, no. 9, pp. 50-57, 2017.

[7] N. Atzei, M. Bartoletti, and T. Cimoli, "A survey of attacks on ethereum smart contracts (SoK)," Lecture Notes in Computer Science, in Proceedings of the International Conference on Principles of Security and Trust, pp. 164-186, Thessaloniki, Greece, April 2017.

[8] F. Idelberger, G. Governatori, R. Riveret, and G. Sartor, "Evaluation of logic-based smart contracts for blockchain systems," in Proceedings of the International Symposium on Rules and Rule Markup Languages for the Semantic Web, pp. 167-183, New York, USA, June 2016.

[9] L. Luu, D. H. Chu, H. Olickel, P. Saxena, and A. Hobor, "Making smart contracts smarter," in Proceedings of the ACM SIGSAC Conference On Computer And Communications Security, pp. 254-269, Vienna, Austria, October 2016.

[10] F. Q. Z. Yao, "BlockChain based supply chain financial risk management research," in Proceedings of the 2020 International Conference On Mechanical Automation And Computer Engineering (MACE), pp. 170-180, Nanchang, China, 2020.

[11] Y. Fu and J. Zhu, "Big production enterprise supply chain endogenous risk management based on blockchain," IEEE Access, vol. 7, no. 8626088, pp. 15310-15319, 2019.

[12] S. K. Dwivedi, R. Amin, and S. Vollala, "Blockchain based secured information sharing protocol in supply chain 
management system with key distribution mechanism," Journal of Information Security and Applications, vol. 54, no. 102554, pp. 1-15, 2020.

[13] E. H. Alfonso-Lizarazo, J. R. Montoya-Torres, and E. G. Franco, "Modeling reverse logistics process in the agroindustrial sector: the case of the palm oil supply chain," Applied Mathematical Modelling, vol. 37, no. 23, pp. 96529664, 2013.

[14] H. Yuan, H. Qiu, Y. Bi, S.-H. Chang, and A. Lam, "Analysis of coordination mechanism of supply chain management information system from the perspective of block chain," Information Systems and E-Business Management, vol. 18, no. 4, pp. 681-703, 2020.

[15] Z. Nehai, P. Piriou, and F. Daumas, "Model-checking of smart contracts," in Proceedings of the IEEE Smart Data (SmartData), pp. 980-987, Halifax, NS, Canada, July 2018.

[16] N. Kshetri, "1 Blockchain's roles in meeting key supply chain management objectives," International Journal of Information Management, vol. 39, pp. 80-89, 2018.

[17] S. E. Chang, Y.-C. Chen, and M.-F. Lu, "Supply chain reengineering using blockchain technology: a case of smart contract based tracking process," Technological Forecasting and Social Change, vol. 144, pp. 1-11, 2019.

[18] I. Ahmed and S. Dixit, "Role of technologies in revamping the supply chain management of kirana stores," Blockchain Applications in IoT Ecosystem, pp. 275-287, EAI/Springer Innovations in Communication and Computing, Europe, 2021.

[19] R. Kamran, N. Khan, and B. Sundarakani, "Blockchain technology development and implementation for global logistics operations: a reference model perspective," Journal of Global Operations and Strategic Sourcing, vol. 14, no. 4, pp. 360-382, 2021.

[20] Z. Liu and P. Guo, "Supply chain decision model based on blockchain: a case study of fresh food E-commerce supply chain performance improvement," Discrete Dynamics in Nature and Society, vol. 2021, Article ID 5795547, 14 pages, 2021.

[21] M. Yoo and Y. Won, "A study on the transparent price tracing system in supply chain management based on blockchain," MDPI AG, vol. 11, p. 4037, 2018. 